\title{
ЯМР КВАДРУПОЛЬНЫХ ЯДЕР ВРАЩАЮЩИХСЯ ПОРОШКОВ: СПЕКТРЫ НЕЦЕНТРАЛЬНЫХ ПЕРЕХОДОВ ПОЛУЦЕЛЫХ И ПРОИЗВОЛЬНЫХ ПЕРЕХОДОВ ЦЕЛЫХ СПИНОВ
}

\author{
(Представил Э. Липпмаа)
}

\section{1. Введение}

ЯМР с магическим вращением образца (ЯМР МВ) является эффективным методом изучения структуры твердых тел, который дает информацию об изотропных химических сдвигах даже в случае квадрупольных ядер. Этот метод, как правило, применялся в случае полуцелых спинов (единственное исключение - дейтерий $\left.\left[{ }^{1}\right]\right)$, причем в основном интерпретировались только линии центрального перехода.

Оказывается, что в ряде случаев для полуцелых спинов целесообразнее использовать в целях повышения точности измерения изотропного химического сдвига не основную линию центрального перехода, а боковые полосы вращения от нецентральных переходов $\left[{ }^{2,3}\right]$. Кроме того, не существует принципиальных трудностей, исключающих применение ЯМР МВ в случае квадрупольных ядер с целыми спинами.

Ясно, что большинство формул в $[4,5,6]$, описывающих форму основной линии центрального перехода полуцелого спина порошкового образца, непосредственно не применимы для интерпретации основных полос нецентральных переходов, боковых полос вращения или любых полос целого спина. Действительно, в отличие от основной полосы центрального перехода в случае первых боковых полос вращения нецентральных переходов спинов $I=\frac{3}{2}$ и $I=\frac{5}{2}$ экспериментально разрешено четыре максимума (см. раздел 3). Различие между формой линий центрального перехода полуцелого спина и всех остальных переходов (включая все переходы целого спина) возникает из-за квадрупольного взаимодействия первого порядка, отсутствующего в первом случае, но сильно влияющего на распределение интенсивностей между основной и множеством боковых полос во втором случае.

В настоящей работе проводится анализ спектра ЯМР MВ квадрупольных ядер порошковых образцов в общем случае, когда распределение интенсивностей между боковыми полосами определяется в основном квадрупольным взаимодействием первого порядка. Выводятся приближенные уравнения, определяющие положения локальных максимумов и центра тяжести линий в зависимости от параметра асимметрии $(\eta)$ тензора градиента электрического поля (ГЭП). Изучается влияние малых изменений частоты вращения образца $\left(\omega_{r}\right)$ на положение центра тяжести линии. 
Положения сингулярных точек, определенные посредством вышенамеченных приближенных формул, хорошо согласуются как с результатами численных расчетов, так и с экспериментально наблюдаемыми положениями их.

\section{2. Частота и интенсивность линии}

В настоящей статье рассматривается случай, когда спин-система монокристалла состоит из одного квадрупольного ядра и учитываются только зеемановский, изотропный химический сдвиг и квадрупольные взаимодействия $\left[{ }^{6}\right]$.

Эволюция состояния спиновой системы описывается эффективным гамильтонианом

$$
\mathbf{H}_{e f f}=\overline{\tilde{\mathbf{H}}}_{Q}^{(0)}+\overline{\tilde{\mathbf{H}}}_{Q}^{(1)}+\overline{\tilde{\mathbf{H}}}_{Q}^{(2)},
$$

рассчитанным во втором приближении теории среднего гамильтониана по периоду Лармора $\left(\omega_{L}^{-1}\right)$. При этом, как и в $\left[{ }^{4,5,6}\right]$, индуцированная вращением временная зависимость вводится параметрически. Такая процедура вполне оправдала себя при теоретической интерпретации экспериментов ЯМР МВ квадрупольных ядер с полуцелыми спинами.

Так как $\overline{\tilde{\mathbf{H}}}_{Q}^{(0)}$ диагонален на базе собственных функций $|m\rangle$ оператора $\mathbf{I}_{Z}\left[{ }^{6}\right]$ и разности $\overline{\tilde{H}}_{Q m, m}^{(0)}-\overline{\tilde{\tilde{H}}}_{Q m-1, m-1}^{(0)}$ имеют нулевые средние значения при магическом значении угла $\theta$ между осью вращения образца и направлением внешнего постоянного магнитного поля $\left(\cos ^{2} \theta=\frac{1}{3}\right)$, то резонансную частоту любого одноквантового перехода $\left(\omega_{m, m-1}\right)$ произвольного спина I во вращающейся с частотой $\omega_{L}$ системе координат можно представить в виде

$$
\omega_{m, m-1}=\Omega_{m, m-1}+\Delta \Omega_{m, m-1}+\sum_{n} a_{m, m-1}^{(n)} \cos n\left(\omega_{r} t-\alpha-\varphi_{m, m-1}^{(n)}\right) .
$$

Здесь $\Omega_{m, m-1}$ учитывает среднее значение сдвига частоты, обусловленного диагональными элементами $\overline{\tilde{H}}_{Q}^{(1)}\left[{ }^{6}\right]$, а слагаемое $\Delta \Omega_{m, m-1}$ есть поправка к среднему значению сдвига частоты, связанная с недиагональными элементами $\overline{\tilde{\mathbf{H}}}_{Q}^{(1)}$ и с диагональными элементами $\overline{\tilde{\mathbf{H}}}_{Q}^{(2)}$.

В случае магического угла первые слагаемые в (1) для симметрических переходов совпадают $\left(\Omega_{m, m-1}=\Omega_{-(m-1),-m}\right)$

$$
\begin{array}{r}
\Omega_{m, m-1}=-\sigma_{m, m-1}\left(1+\frac{1}{3} \eta^{2}\right) \frac{\omega_{Q}}{\omega_{L}} \omega_{Q}+ \\
+\frac{2}{9} N_{m, m-1} \frac{\omega_{Q}}{\omega_{L}} \omega_{Q}\left[B^{2}+C^{2}+2\left(D^{2}+F^{2}\right)\right] .
\end{array}
$$

Здесь коэффициенты сдвига $\sigma_{m, m-1}$ и ширины линий $N_{m, m-1}$ определяются следующими выражениями:

$$
\begin{aligned}
\sigma_{m, m-1} & =\frac{1}{6}[2 I(I+1)-14 m(m-1)-5], \\
N_{m, m-1} & =\frac{1}{6}[6 I(I+1)-34 m(m-1)-13] .
\end{aligned}
$$


Остальные обозначения в (2) совпадают с соответствующими обозначениями в $[4,6]$, т. е.

$$
\begin{gathered}
\omega_{Q}=\frac{3 e^{2} Q q}{4 I(2 I-1) \hbar}, \\
A=\frac{1}{4}\left[\left(1-3 \cos ^{2} \beta\right)-\eta \sin ^{2} \beta \cos 2 \gamma\right], \\
B=\frac{1}{4}\left[3 \sin ^{2} \beta+\eta\left(1+\cos ^{2} \beta\right) \cos 2 \gamma\right], \\
C=\frac{1}{2} \eta \cos \beta \sin 2 \gamma, \\
D=\frac{1}{4} \sin 2 \beta(3-\eta \cos 2 \gamma), \\
F=\frac{1}{2} \eta \sin \beta \sin 2 \gamma,
\end{gathered}
$$

a $\alpha, \beta, \gamma$ - эйлеровы углы, определяющие ориентацию системы собственных осей тензора ГЭП относительно системы координат, связанной с ротором.

При учете влияния недиагональных элементов $\overline{\tilde{\mathbf{H}}}_{Q}^{(1)}$ в первом приближении теории возмущений второе слагаемое в выражении (2) принимает следующий вид:

$$
\begin{aligned}
\Delta \Omega_{m, m-1} & =\frac{1}{18}\left(\frac{\omega_{Q}}{\omega_{L}}\right)^{2} \omega_{Q}(2 m-1) \times \\
& \times\left\{\overline{V_{-1} V_{0} V_{1}}[-12 I(I+1)+40 m(m-1)+27]+\right. \\
& +\overline{V_{-2} V_{0} V_{2}}[6 I(I+1)-10 m(m-1)-12]+ \\
& \left.+\frac{1}{2} \overline{\left(V_{-1} V_{2} V_{-1}+V_{1} V_{-2} V_{1}\right)}[-8 I(I+1)+20 m(m-1)+15]\right\},
\end{aligned}
$$

где через $V_{i}, i=0, \pm 1, \pm 2$ обозначены компоненты тензора ГәП в лабораторной системе координат (см. уравнения (5) в [6]), а черта над выражением означает среднее значение.

В случае характерного для легких и средних ядер умеренного квадрупольного взаимодействия

$$
\left|\omega_{L}\right| \gg\left|\omega_{Q}\right| \gg\left|\omega_{r}\right|,
$$

опишем вначале детектируемые спектральные линии в приближении, в котором не учитывается член $\overline{\tilde{\mathbf{H}}}_{Q}^{(2)}$ и недиагональные элементы $\overline{\tilde{\mathbf{H}}}_{Q}^{(1)}$. Влияние этих членов эффективного гамильтониана рассмотрено в последнем параграфе настоящей статьи.

В принятом приближении из (1) с очевидностью следует (отметим, что в этом случае индекс суммирования принимает значения $n=1,2,3,4)$, что любая пара симметрических переходов каждого индивидуального кристаллика порошкового образца индуцирует в спектре ЯМР МВ множество боковых полос на частотах

$$
\Omega_{m, m-1}+s \omega_{r}, \quad s=0, \pm 1 \pm 2, \ldots,
$$


положения которых определены выражением (2). При этом первое слагаемое в (2) характеризует постоянный, не зависящий от ориентации монокристалла квадрупольный сдвиг частоты линии от Ларморовской частоты. $\sigma_{m, m-1}$ - константа, зависящая от величины спина и рассматриваемого перехода. Зависящий от ориентации компонент квадрупольного сдвига учитывается вторым слагаемым в уравнении (2). Для порошкового образца этот член определяет пределы отличной от нуля интенсивности как основной линии $(s=0)$, так и боковых полос вращения $(s \neq 0)$.

Таблища 1

Коэффициенты перехода полуцелых спинов

\begin{tabular}{c|c|c|c|c|c}
\hline$I$ & $m$ & $\sigma_{m, m-1}$ & $N_{m, m-1}$ & $N_{m, m-1}^{(1)}$ & $N_{m, m-1}^{(2)}$ \\
\hline \multirow{2}{*}{$3 / 2$} & $1 / 2$ & 1 & 3 & 4 & 2 \\
& $3 / 2$ & $-4 / 3$ & $-8 / 3$ & $-8 / 3$ & $-8 / 9$ \\
$5 / 2$ & $1 / 2$ & $8 / 3$ & 8 & $32 / 3$ & $16 / 3$ \\
& $3 / 2$ & $1 / 3$ & $7 / 3$ & 4 & $22 / 9$ \\
& $5 / 2$ & $-20 / 3$ & $-44 / 3$ & -16 & $-56 / 9$ \\
$7 / 2$ & $1 / 2$ & 5 & 15 & 20 & 10 \\
& $3 / 2$ & $8 / 3$ & $28 / 3$ & $40 / 3$ & $64 / 9$ \\
& $5 / 2$ & $-13 / 3$ & $-23 / 3$ & $-20 / 3$ & $-14 / 9$ \\
$9 / 2$ & $7 / 2$ & -16 & -36 & -40 & -16 \\
& $1 / 2$ & 8 & 24 & 32 & 16 \\
& $3 / 2$ & $17 / 3$ & $55 / 3$ & $76 / 3$ & $118 / 9$ \\
& $5 / 2$ & $-4 / 3$ & $4 / 3$ & $16 / 3$ & $40 / 9$ \\
& $7 / 2$ & -13 & -27 & -28 & -10 \\
& $9 / 2$ & $-88 / 3$ & $-200 / 3$ & $-224 / 3$ & $-272 / 9$
\end{tabular}

таблица 2

Коэффициенты перехода целых спинов

\begin{tabular}{|c|c|c|c|c|c|}
\hline$I$ & $m$ & $\sigma_{m, m-1}$ & $N_{m, m-1}$ & $N_{m, m-1}^{(1)}$ & $N_{m, m-1}^{(2)}$ \\
\hline 1 & 1 & $-1 / 6$ & $-1 / 6$ & 0 & $1 / 9$ \\
\hline 2 & $\begin{array}{l}1 \\
2\end{array}$ & $\begin{array}{r}7 / 6 \\
-7 / 2\end{array}$ & $\begin{array}{r}23 / 6 \\
-15 / 2\end{array}$ & $\begin{array}{l}16 / 3 \\
-8\end{array}$ & $\begin{array}{l}25 / 9 \\
-3\end{array}$ \\
\hline 3 & $\begin{array}{l}1 \\
2 \\
3\end{array}$ & $\begin{array}{r}19 / 6 \\
-3 / 2 \\
-65 / 6\end{array}$ & $\begin{array}{r}59 / 6 \\
-3 / 2 \\
-145 / 6\end{array}$ & $\begin{array}{c}40 / 3 \\
0 \\
-80 / 3\end{array}$ & $\begin{array}{c}61 / 9 \\
1 \\
-95 / 9\end{array}$ \\
\hline 4 & $\begin{array}{l}1 \\
2 \\
3 \\
4\end{array}$ & $\begin{array}{r}35 / 6 \\
7 / 6 \\
-49 / 6 \\
-133 / 6\end{array}$ & $\begin{array}{r}107 / 6 \\
39 / 6 \\
-97 / 6 \\
-301 / 6\end{array}$ & $\begin{array}{c}24 \\
32 / 6 \\
-16 \\
-56\end{array}$ & $\begin{array}{r}109 / 9 \\
57 / 9 \\
-47 / 9 \\
-203 / 9\end{array}$ \\
\hline 5 & $\begin{array}{l}1 \\
2 \\
3 \\
4 \\
5\end{array}$ & $\begin{array}{r}55 / 6 \\
9 / 2 \\
-29 / 6 \\
-113 / 6 \\
-75 / 2\end{array}$ & $\begin{array}{r}167 / 6 \\
33 / 2 \\
-37 / 6 \\
-241 / 6 \\
-171 / 2\end{array}$ & $\begin{array}{c}112 / 3 \\
24 \\
-8 / 3 \\
-128 / 3 \\
-96\end{array}$ & $\begin{array}{c}169 / 9 \\
13 \\
13 / 9 \\
-143 / 9 \\
-39\end{array}$ \\
\hline 6 & $\begin{array}{l}1 \\
2 \\
3 \\
4 \\
5 \\
6\end{array}$ & $\begin{array}{r}79 / 6 \\
17 / 2 \\
-5 / 6 \\
-89 / 6 \\
-67 / 2 \\
-341 / 6\end{array}$ & $\begin{array}{r}239 / 6 \\
57 / 2 \\
35 / 6 \\
-169 / 6 \\
-147 / 2 \\
-781 / 6\end{array}$ & $\begin{array}{c}160 / 3 \\
40 \\
40 / 3 \\
-80 / 3 \\
-80 \\
-440 / 3\end{array}$ & $\begin{array}{c}241 / 9 \\
21 \\
85 / 9 \\
-71 / 9 \\
-31 \\
-539 / 9\end{array}$ \\
\hline
\end{tabular}


Коэффициенты перехода $\sigma_{m, m-1}$ и $N_{m, m-1}$ позволяют грубо оценить изотропный химический сдвиг квадрупольного ядра из спектров ЯМР MB. Обратим внимание на то, что до значений $I=\frac{17}{2}$ в случае полуцелых и до $I=5$ в случае целых спинов минимальное значение коэффициента $\sigma_{m, m-1}$ соответствует двукратно симметрическому переходу $m_{2 s} \leftrightarrow m_{2 s}-1$, если только такое для данного ядра существует $\left(m_{2 s}=n+\frac{1}{2}\right.$ и $m_{2 s}=n+1, n=1,2, \ldots$, в случае полуцелого $I=2 n+\frac{1}{2}$ и целого $I=2 n+1$ спинов соответственно; табл. 1,2$)$. Одновременно имеет минимальное значение также и коэффициент полной ширины этой линии $N_{m, m-1}$.

Очевидно, что большой практический интерес представляют переходы $3 / 2 \leftrightarrow 1 / 2,5 / 2 \leftrightarrow 3 / 2$ и $2 \leftrightarrow 1$ спинов $I=5 / 2, I=9 / 2$ и $I=3$ соответственно.

Далее, с целью более детального анализа форм линий порошкового образца целесообразно выразить положения линии одного выбранного монокристаллика через безразмерную приведенную частоту $f$, определенную как

$$
f=\frac{\Omega_{m, m-1}+\sigma_{m, m-1}\left(1+\frac{1}{3} \eta^{2}\right) \frac{\omega_{Q}}{\omega_{L}} \omega_{Q}}{N_{m, m-1} \frac{\omega_{Q}}{\omega_{L}} \omega_{Q}},
$$

которая не зависит от параметров конкретного перехода. С помощью выражений (7)-(10) получаем

$$
\begin{gathered}
f=\frac{1}{18}\left[\eta^{2}-\left(9-\eta^{2}\right) \sin ^{2} \beta+9 \sin ^{2} \beta(3-\eta \cos 2 \gamma)-\right. \\
\left.-\frac{7}{4} \sin ^{4} \beta(3-\eta \cos 2 \gamma)^{2}\right] .
\end{gathered}
$$

Таким образом, положения линий одного произвольного монокристаллика порошкового образца зависят только от двух эйлеровых углов $\beta$ и $\gamma$, определяющих его ориентацию относительно оси ротора.

Интенсивность линий одного монокристаллика в спектре зависит от полного набора эйлеровых углов $\alpha, \beta, \gamma$, и симметрические переходы дают в (1) равные амплитуды и фазы только для третьей и четвертой гармоник даже в случае точной настройки магического угла. Действительно, амплитуды и фазы определены соответственно выражениями

$$
\begin{gathered}
a_{m, m-1}^{(n)}=\left\{\left[b_{m, m-1}^{(n)}\right]^{2}+\left[c_{m, m-1}^{(n)}\right]^{2}\right\}^{1 / 2} \\
n \varphi_{m, m-1}^{(n)}=\arctan \frac{b_{m, m-1}^{(n)}}{c_{m, m-1}^{(n)}}
\end{gathered}
$$

где

$$
\begin{aligned}
& b_{m, m-1}^{(1)}=-\frac{2 \sqrt{2}}{3} \omega_{Q}\left\{(2 m-1) D+\frac{\omega_{Q}}{\omega_{L}}\left[N_{m, m-1}^{(1)} A D-N_{m, m-1}^{(2)}(B D-F C)\right]\right\}, \\
& c_{m, m-1}^{(1)}=-\frac{2 \sqrt{2}}{3} \omega_{Q}\left\{(2 m-1) F+\frac{\omega_{Q}}{\omega_{L}}\left[N_{m, m-1}^{(1)} A F+N_{m, m-1}^{(2)}(B F+C D)\right]\right\}, \\
& b_{m, m-1}^{(2)}=-\frac{2}{3} \omega_{Q}\left\{(2 m-1) C-\frac{\omega_{Q}}{\omega_{L}}\left[2 N_{m, m-1}^{(1)} A C-\frac{2}{9}(2 m-1)^{2} D F\right]\right\},
\end{aligned}
$$




$$
\begin{aligned}
c_{m, m-1}^{(2)}= & -\frac{2}{3} \omega_{Q}\left\{(2 m-1) B-\frac{\omega_{Q}}{\omega_{L}}\left[2 N_{m, m-1}^{(1)} A B+\right.\right. \\
& \left.\left.+\frac{1}{9}(2 m-1)^{2}\left(D^{2}-F^{2}\right)\right]\right\}, \\
b_{m, m-\not}^{(3)}= & \frac{4 \sqrt{2}}{9} \frac{\omega_{Q}}{\omega_{L}} \omega_{Q} N_{m, m-1}(B D+C F), \\
c_{m, m-1}^{(3)}= & -\frac{4 \sqrt{2}}{9} \frac{\omega_{Q}}{\omega_{L}} \omega_{Q} N_{m, m-1}(C D-B F), \\
b_{m, m-1}^{(4)}= & \frac{4}{9} \frac{\omega_{Q}}{\omega_{L}} \omega_{Q} N_{m, m-1} B C, \\
c_{m, m-1}^{(4)}= & -\frac{2}{9} \frac{\omega_{Q}}{\omega_{L}} \omega_{Q} N_{m, m-1}\left(C^{2}-B^{2}\right) .
\end{aligned}
$$

Так как значения коэффициентов

$$
\begin{aligned}
& N_{m, m-1}^{(1)}=\frac{4}{3}[I(I+1)-5 m(m-1)-2], \\
& N_{m, m-1}^{(2)}=\frac{1}{9}[6 I(I+1)-26 m(m-1)-11]
\end{aligned}
$$

не изменяются при подстановке - $(m-1)$ вместо $m$, то ясно, что $a_{m, m-1}^{(n)}$ и $\varphi_{m, m-1}^{(n)} \quad$ несколько отличаются от $a_{-(m-1),-m}^{(n)}$ и $\varphi_{-(m-1),-m}^{(n)}$ в случаях $n=1,2$.

Интенсивность спектральных линий одного монокристалла можно выразить через Бесселовы функции первого порядка $J_{n}(x)$ [7], а суммарная интенсивность $I_{m, m-1}^{(s)}(\beta, \gamma)$ на частоте $f(\beta, \gamma)$ в $s$-й боковой полосе, обеспечиваемая переходами $m \leftrightarrow m-1$ всех монокристаллов образца с двумя фиксированными углами $\beta$ и $\gamma$, пропорциональна величине

$$
\left\{\left[A_{m, m-1}^{(s)}(\beta, \gamma)\right]^{2}+\left[B_{m, m-1}^{(s)}(\beta, \gamma)\right]^{2}\right\} \sin \beta
$$

где

$$
\begin{aligned}
A_{m, m-1}^{(s)} & =\sum_{l} \sum_{k} \sum_{n} J_{l}\left(\frac{a^{(4)}}{4 \omega_{r}}\right) J_{k}\left(\frac{a^{(3)}}{3 \omega_{r}}\right) J_{n}\left(\frac{a^{(2)}}{2 \omega_{r}}\right) J_{s-2 n-3 k-4 l}\left(\frac{a^{(1)}}{\omega_{r}}\right) \times \\
& \times \cos \left[(s-2 n-3 k-4 l) \varphi^{(1)}+2 n \varphi^{(2)}+3 k \varphi^{(3)}+4 l \varphi^{(4)}\right], \\
B_{m, m_{\gamma}-1}^{(s)} & =\sum_{l} \sum_{k} \sum_{n} J_{l}\left(\frac{a^{(4)}}{4 \omega_{r}}\right) J_{k}\left(\frac{a^{(3)}}{3 \omega_{r}}\right) J_{n}\left(\frac{a^{(2)}}{2 \omega_{r}}\right) J_{s-2 n-3 k-4 l}\left(\frac{a^{(1)}}{\omega_{r}}\right) \times \\
& \times \sin \left[(s-2 n-3 k-4 l) \varphi^{(1)}+2 n \varphi^{(2)}+3 k \varphi^{(3)}+4 l \varphi^{(4)}\right] .
\end{aligned}
$$

Здесь в целях сокращения записи индексы перехода $m, m-1$ при $a_{m, m-1}^{(n)}$ и $\varphi_{m, m-1}^{(n)}$ не повторяются.

При выводе выражений (20)-(22) предполагалось случайное распределение монокристаллов в образце и интегрирование проводилось по углу $\alpha$.

Симметрический переход, $-(m-1) \leftrightarrow-m$ также дает свой вклад в интенсивность $I_{\rightarrow(m-1),-m}^{(s)}(\beta, \gamma)$ на частоте $f(\beta, \gamma) s$-й боковой полосы. Этот вклад несколько отличается от вклада $I_{m, m-1}^{(s)}(\beta, \gamma)$ и может быть 
определен из $(20)-(22)$ посредством подстановки $a_{-(m-1),-m}^{(n)}$ и $\varphi_{-(m-1),-m}^{(n)}$ вместо $a_{m, m-1}^{(n)}$ и $\varphi_{m, m-1}^{(n)}$.

Таким образом, распределение интенсивности в $s$-й боковой полосе, т. е. формы линий боковых полос описываются в общем случае очень сложными формулами. Простые аналитические выражения для истинной формы линий боковых полос отсутствуют, и численные расчеты трудоемки. Поэтому желательно проанализировать возможности упрощения выражений распределения интенсивности. Из уравнений (15)(17) видно, что существенное упрощение достигается в случае, когда все коэффициенты перехода (4), (18) и (19) (обозначены ниже как $N)$ не слишком велики

$$
\left|N \frac{\omega_{Q}^{2}}{\omega_{L} \omega_{T}}\right| \ll 1,
$$

Действительно, в этом случае, во-первых, в (21) и (22) отпадает суммирование по индексам $k$ и $l$. Во-вторых, обосновано пренебрежение в (17) слагаемыми, пропорциональными $\frac{\omega_{Q}}{\omega_{L}}$ при определении аргументов двух оставшихся в (21) и (22) Бесселовых функций. При этом обнаруживается равенство вкладов интенсивностей от симметрических переходов $m \leftrightarrow m-1$ и $-(m-1) \leftrightarrow-m$ монокристалла.

С учетом (23) компоненты (20) $A_{m, m-1}^{(s)}$ и $B_{m, m-1}^{(s)}$ могут быть переписаны следующим образом:

$$
\begin{gathered}
A_{m, m-1}^{(s)}=J_{0}\left(\frac{a^{(2)}}{2 \omega_{r}}\right) J_{s}\left(\frac{a^{(1)}}{\omega_{r}}\right)+ \\
+\sum_{n=1}^{\infty} J_{n}\left(\frac{a^{(2)}}{2 \omega_{r}}\right)\left[(-1)^{s} J_{2 n-s}\left(\frac{a^{(1)}}{\omega_{r}}\right)+(-1)^{n} J_{2 n+s}\left(\frac{a^{(1)}}{\omega_{r}}\right)\right] \cos 2 n\left(\varphi^{(2)}-\varphi^{(1)}\right),
\end{gathered}
$$

$$
\begin{aligned}
& B_{m, m-1}^{(s)}=\sum_{n=1}^{\infty} J_{n}\left(\frac{a^{(2)}}{2 \omega_{r}}\right)\left[(-1)^{s} J_{2 n-s}\left(\frac{a^{(1)}}{\omega_{r}}\right)-\right. \\
& \left.-(-1)^{n} J_{2 n+s}\left(\frac{a^{(1)}}{\omega_{r}}\right)\right] \sin 2 n\left(\varphi^{(2)}-\varphi^{(1)}\right) .
\end{aligned}
$$

Отметим, что настоящее приближение неприменимо в случае изучения распределения интенсивностей в спектре центрального перехода $(m=1 / 2)$ полуцелого спина. В этом случае боковые полосы возникают только благодаря квадрупольному взаимодействию второго порядка $\left(\overline{\tilde{\mathbf{H}}}_{Q}^{(1)}\right)$. Основанием для настоящего приближения является принятое в настоящей работе предположение о формировании боковых полос посредством только квадрупольного взаимодействия первого порядка $\left(\overline{\tilde{H}}_{Q}^{(0)}\right)$.

Хотя среди переходов более распространенных значений $I$ имеется, как правило, переход, для которого $\left|N_{\max }\right|$ не превышает 10 , все же условия (23) в ряде практических случаев выполняются не очень хорошо. Так, например, даже в поле 11,7 Тесла при скорости вращения 


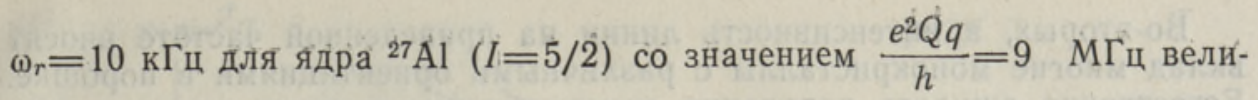
чина $\left|N \frac{\omega_{Q}^{2}}{\omega_{L} \omega_{r}}\right|=|0,35 N|$.

Следует заметить, что аргументами Бесселовых функций являются не величины (23), а произведение трех сомножителей-величин, задаваемых (23), и двух функций, зависящих от ориентации $(6)-(10)$. Абсолютные значения этих функций в общем случае «1 и имеют порядок 1 лишь в сравнительно узком интервале ориентаций монокристалла.

Точные значения интенсивностей боковых полос обычно не требуются. В практических задачах, таких как определение изотропного химического сдвига и параметров квадрупольного взаимодействия, сделанное приближение является вполне удовлетворительным и совпадение между численными расчетами и экспериментальными формами линий оказывается хорошим.

Естественно, чем выше напряженность постоянного магнитного поля и частота вращения и чем меньше константа квадрупольного взаимодействия, тем это приближение лучше.

\section{3. Форма линии и центр тяжести боковых полос}

Симметрические переходы всех монокристаллов образца с двумя фиксированными углами ориентации $\beta$ и $\gamma$ индуцируют одну линию в $s$-й боковой полосе спектра ЯМР МВ. Как следует из выражений (14), $(20)$, а также (24) и (25), положение и интенсивность линий сложным образом зависят от ориентации монокристалла, от параметров квадрупольного взаимодействия и от условий проведения эксперимента. Поэтому точный алгебраический анализ формы боковых полос крайне неудобен.

Однако качественный анализ отмеченных выше уравнений дает возможность выявить положение локальных максимумов в зависимости от величины $\eta$.

Во-первых, из уравнений (24), (25) видно, что максимальные значения слагаемых $A_{m, m-1}^{(s)}$ и $B_{m, m-1}^{(s)}$ следует ожидать для первых боковых полос (в случае малых $|s|$ ) при таких ориентациях, для которых аргументы Бесселовых функций удовлеворяют условию

$$
a_{m, m-1}^{(1)}=a_{m, m-1}^{(2)} .
$$

Это условие вместе с дополнительным требованием $\sin 2 \gamma=0$ (при котором $\cos 2 n\left(\varphi^{(2)}-\varphi^{(1)}\right)$ принимает значения \pm 1$)$, дает основание ожидать появления локальных максимумов на линии боковых полос в положениях

$$
\begin{aligned}
& f_{1}=\frac{4}{81}[2-(1-\eta)(\eta+2 \sqrt{1+\eta})] \\
& f_{2}=\frac{4}{81}[2+(1+\eta)(\eta-2 \sqrt{1-\eta})] \\
& f_{3}=\frac{4}{81}[2-(1-\eta)(\eta-2 \sqrt{1+\eta})] \\
& f_{4}=\frac{4}{81}[2+(1+\eta)(\eta+2 \sqrt{1-\eta})]
\end{aligned}
$$


Во-вторых, в интенсивность линии фа приведенной частоте вносят вклад многие монокристаллы с различными ориентациями в порошке. Естественно ожидать появления локальных максимумов при тех значениях $f$, для которых эти интервалы углов ориентации являются наибольшими. Из (14) вытекает, что отмеченные $\beta$-интервалы ограничены кривыми $\left(y=\sin ^{2} \beta\right)$

$$
\begin{aligned}
& y_{+,-}=\frac{18}{7(3 \pm \eta)}, \\
& y_{0}=\frac{1}{7\left(9-\eta^{2}\right)}\left[81+7\left(\eta^{2}-18 f\right)\right], \\
& y_{1,2}=\frac{2}{7} \frac{6+\eta \pm \sqrt{(6+\eta)^{2}+7\left(\eta^{2}-18 f\right)}}{3+\eta}, \\
& y_{3,4}=\frac{2}{7} \frac{6-\eta \pm \sqrt{(6-\eta)^{2}+7\left(\eta^{2}-18 t\right)}}{3-\eta} .
\end{aligned}
$$

Рис. 1 и 2 вместе с уравнениями (27) наталкивают на мысль о том, что локальные максимумы линий боковых полос следует ожидать при

$$
f_{5}= \begin{cases}\frac{1}{8}\left(1+\frac{1}{3} \eta\right)^{2}, & \eta \leqslant \frac{3}{7} \\ \frac{1}{7}+\frac{1}{9} \eta^{2}, & \eta \geqslant \frac{3}{7}\end{cases}
$$

и

$$
f_{6}=\frac{1}{18}\left[\eta^{2}+\frac{1}{7}(6-\eta)^{2}\right]
$$

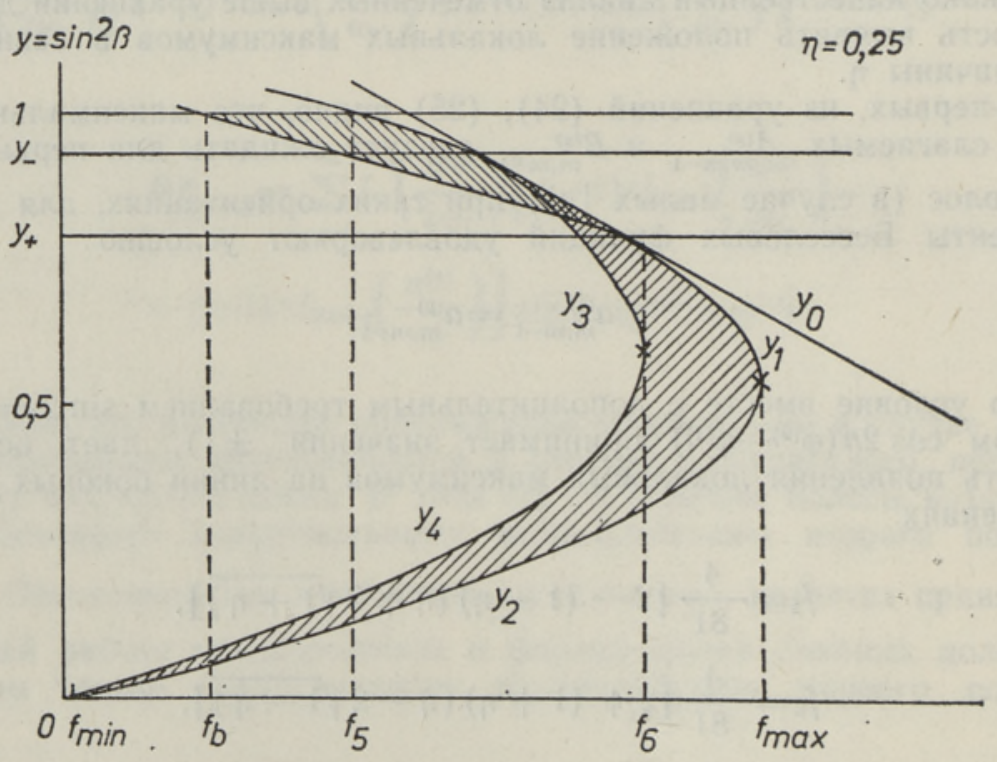

Рис. 1. Вид области определения ориентационного угла $\beta$ монокристаллов образца, вносящих вклад в интенсивность спектральной линии порошка на фиксированной частоте $f$ для случая $\eta \leqslant \frac{3}{7}$. 


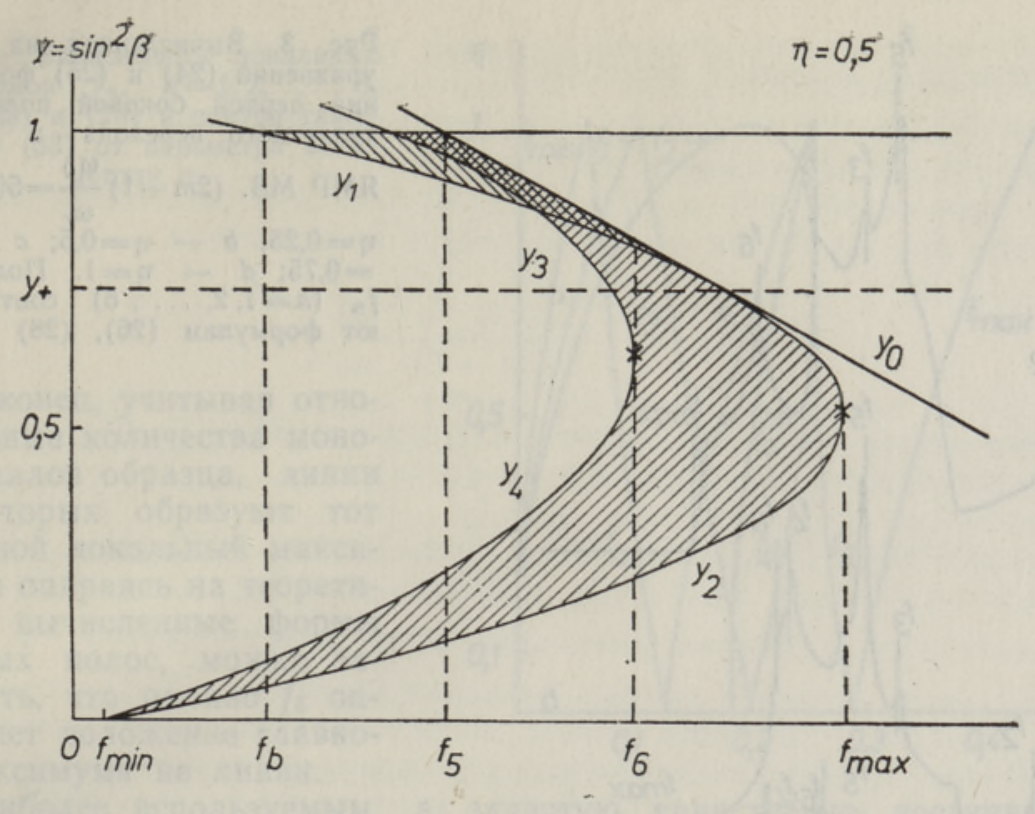

Рис. 2. Вид области определения ориентационного угла $\beta$ монокристаллов образца, вносящих вклад в интенсивность спектральной линии порошка на фиксированноі: частоте $f$ для случая $\eta \geqslant \frac{3}{7}$.

Кроме того, из уравнений (27) для полной ширины линий имеем

$$
\begin{aligned}
& f_{\min }=\frac{1}{18} \eta^{2} \\
& f_{\max }=\frac{1}{18}\left[\eta^{2}+\frac{1}{7}(6+\eta)^{2}\right] .
\end{aligned}
$$

Численные расчеты показывают (рис. 3), что выражения (26), (28) и (29) достаточно хорошо предсказывают положения локальных максимумов на теоретической форме линии.

Зарегистрированная экспериментально $\left[{ }^{2}\right]$ форма линии первой боковой полосы сателлитного перехода, $m=3 / 2,{ }^{23} \mathrm{Na}$ в $\mathrm{NaNO}_{2}\left(\frac{e^{2} Q q}{\hbar}=1,1\right.$ МГц, $\eta=0,1)$ (рис. 4) указывает на удовлетворительное согласие между положениями выявленных экспериментально и предсказанных на основе $(26),(28)$ и (29) сингулярных точек. Действительно, в эксперименте получены максимумы на частотах $v^{\prime}=2075$ Гц, $v_{5}=1635$ Гц, $v^{\prime \prime}=1464$ Гц и $v_{6}=1223$ Гц. В случае $\eta=0,1$ в качестве теоретических значений, определяющих положения этих максимумов, следует принимать (см. рис. 5) соответственно выражения $f^{\prime}=\frac{1}{2}\left(f_{1}+f_{2}\right)$, $f_{5}, f^{\prime \prime}=\frac{1}{2}\left(f_{3}+f_{4}\right)$ и $f_{6}$. Теперь легко проверить, что значения отношений $\frac{v^{\prime \prime}-v^{\prime}}{v_{5}-v^{\prime}} \frac{f_{5}-f^{\prime}}{f^{\prime \prime}-f^{\prime}}$ и $\frac{v_{6}-v^{\prime}}{v_{5}-v^{\prime}} \frac{f_{5}-f^{\prime}}{f_{6}-f^{\prime}}$ не различаются от 1 больше, чем на $8 \%$. 


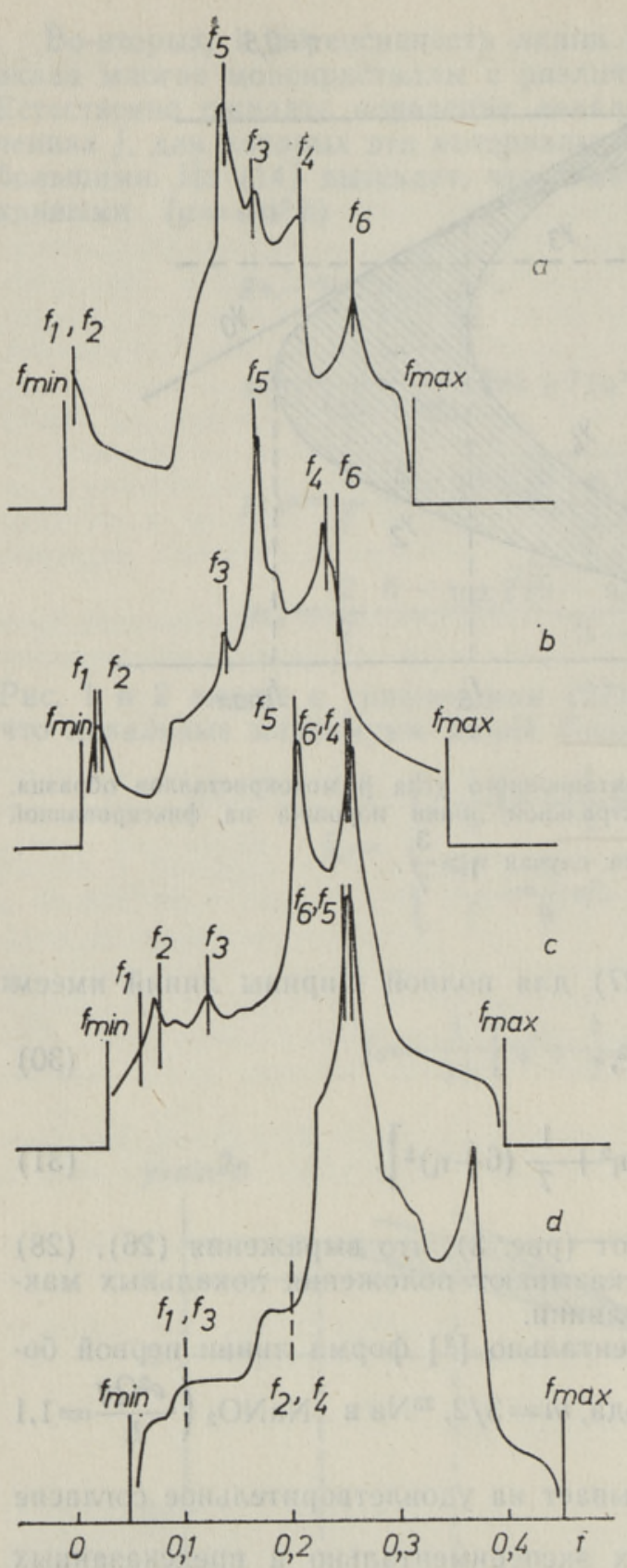

Рेис. 3. В̈численная на̂ основѐ уравнений $(24)$ и $(25)$ форма линии первой боковой полосы сателлитного перехода в спектре ЯMP MB. $(2 m-1) \frac{\omega_{Q}}{\omega_{r}}=500 ; a-$ $\eta=0,25 ; b-\eta=0,5 ; c-\eta=$ $=0,75 ; d-\eta=1$. Положения $f_{n}(n=1,2, \ldots, 6)$ соответствуют формулам (26), (28) и (29).

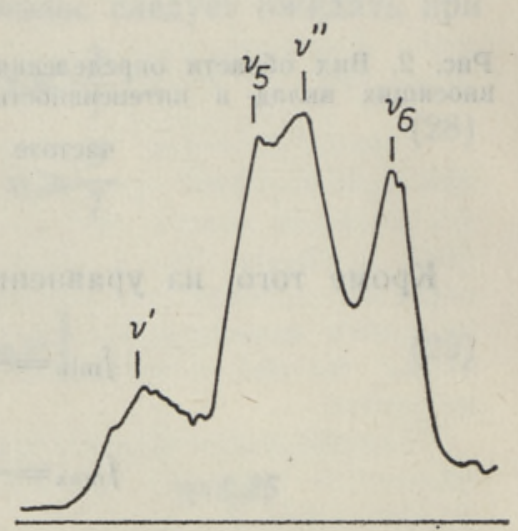

Рис. 4. Экспериментальная первая боковая полоса $\pm \frac{3}{2} \leftrightarrow \pm \frac{1}{2}$ переходов ${ }^{23} \mathrm{Na}$ в $\mathrm{NaNO}_{2}$. Постоянное магнитное поле 4,7 Тесла; $\frac{e^{2} Q q}{h}=1,1$ МГц; $\eta=0,1 ; \frac{\omega_{r}}{2 \pi}=$ $=3967$ Гц. Положения макснмумов $v^{\prime}=2075 \Gamma ц, v_{5}=1635 \Gamma ц$, $v^{\prime \prime}=1464$ Гц и $v_{6}=1223$ Гц.

Следует заметить, что если ошибка в определении частот составляет \pm 10 Гц, то ошибка отношения $\frac{v^{\prime \prime}-v^{\prime}}{v_{5}-v^{\prime}}$ составляет $8 \%$.

Хотя $f_{1}$ и $f_{2}$ располагаются в области линии, где общая интенсивность сравнительно мала $(\sin \beta<0,1)$, все же в реальных спектрах соответствующий им максимум наблюдается достаточно легко. Очевидно, случаи малых или средних значений $\eta$ (приблизительно совпадают $f_{3}$ и $f_{4}$ или $f_{4}$ и $f_{6}$ соответственно, а $f_{5}$ еще достаточно удален от них - см. рис. 5) более выгодны для экспериментального разрешения максимумов в главной области линии. 
Рис. 5. Зависимость локальных максимумов $f_{n}, n=1,2, \ldots, 6$, $(26),(28)$ и $(29)$ и центра тяжести $f_{c}^{(s)}$ (33) от параметра асимметрии $\eta$.

Наконец, учитывая относительные количества монокристаллов образца, линии от которых образуют тот или иной локальный максимум, и опираясь на теоретически вычисленные формы боковых полос, можно заключить, что именно $f_{5}$ определяет положение главного максимума на линин.

Наиболее используемым, а зачастую единственно доступным экспериментально определяемым параметром, позволяющим различать квадрупольный и химический сдвиги линий, является центр тяжести боковой полосы.

Необходимые интегрирования с использованием полных функций $I^{(s)}(\beta, \gamma)$ и $f(\beta, \gamma)$ с целью получения точного алгебраического выражения для центра тяжести $f_{c}^{(s)}$ провести не удалось. Однако получены приближенные уравнения для $f_{c}^{(8)}$.

Из $\left[{ }^{3}\right]$ ясно, что расчет в предположении $I(\beta, \gamma)$ пропорциональна $\sin \beta$ дает выражения для центра тяжести $f_{c}$ всего спектра (основная линия + все боковые полосы) рассматриваемого перехода

$$
f_{c}=\frac{1}{5}\left(1+\frac{1}{3} \eta^{2}\right) \text {. }
$$

Учитывая кажущуюся грубость предположения, сделанного при выводе выражения (32) в случае боковой полосы, следует подчеркнуть, что расхождения между результатами на основе (32) и расчетами не превышает $10 \%$ от полной ширины линии (при $|s|=0,1$ и 2).

Рассчитанные теоретические формы линий подсказывают идею аппроксимировать распределение интенсивности линии боковой полосы треугольником с вершинами $\left(f_{b}, 0\right),\left(f_{5}, I\right)$ и $\left(f_{\max }, 0\right)$. Здесь $f_{b}-$ минимальная частота в спектре, соответствущая ориентации монокристалликов $\beta=90^{\circ}$ (см. рис. 1,2 ).

$$
f_{b}=\frac{1}{8}\left(1-\frac{1}{3} \eta\right)^{2}
$$

Для центра тяжести получаем теперь выражения

$$
f_{c}^{(s)}= \begin{cases}\frac{1}{21}\left(\frac{15}{4}+\frac{2}{3} \eta+\frac{23}{36} \eta^{2}\right), & \eta \leqslant \frac{3}{7} \\ \frac{1}{21}\left(\frac{31}{8}+\frac{1}{12} \eta+\frac{95}{72} \eta^{2}\right), & \eta \geqslant \frac{3}{7} .\end{cases}
$$

Предсказания на основе (33) согласуются с численными расчетами несколько лучше (в пределах $5 \%$ от полной ширины линии). При рассмотрении уравнений $(24)$ и $(25)$ встает вопрос о зависимости $f_{c}^{(8)}$ 
от конкретных значений величины $\frac{\omega_{Q}}{\omega_{r}}$, хотя численные расчеты позволяют утверждать, что если это отношение достаточно большое (превышает 10), то зависимость слабая.

Из выражений (24) и (25) следует, что если изменение частоты вращения $\Delta \omega_{r}$ мало́, так что выполняется соотношение

$$
\left|\Delta \omega_{r}\right|<\left|\frac{1}{2(2 m-1)} \frac{\omega_{r}^{2}}{\omega_{Q}}\right|
$$

то абсолютная величина соответствующего изменения интенсивности достаточно хорошо описывается выражением

$$
\left|\Delta I^{(s)}(\beta, \gamma)\right|=\left|\sqrt{10}(2 m-1) \frac{\omega_{Q}}{\omega_{r}^{2}} \Delta \omega \sqrt{f(\beta, \gamma)} I^{(s)}(\beta, \gamma)\right| .
$$

Следовательно, изменение интенсивности на фиксированной $f$ в спектральной линии порошка пропорционально величине $\sqrt{f} I^{(s)}(f)$, если изменения интенсивности линий от всех монокристаллов имеют одинаковый знак. Далее, для оценки изменения положения центра тяжести используется аппроксимация треугольником, аналогично случаю (33). Чтобы при этом не уменьшать пределы возможного изменения $f_{c}{ }^{(s)}$, изменение интенсивности учитывается только в интервале частот от $f_{5}$ до $f_{\max }$. Описанный способ оценки приводит к следующему выражению для $\Delta f_{c}^{(s)}$ - предела изменения положения центра тяжести, вызванного малым изменением частоты вращения.

$$
\left|\Delta f_{c}^{(s)}\right|=\left|\sqrt{\frac{8}{5}}(2 m-1) \frac{\omega_{Q}}{\omega_{r}^{2}} \Delta \omega_{r}\left(\frac{9}{14} f_{\max }-f_{c}^{(s)}\right)\right| \ldots
$$

Такая оценка допускает изменение положения $f_{c}^{(s)}$ на величину $<9 \%$ от собственного значения, что практически согласуется с численно определенной точностью выражений (33).

В заключение этого параграфа отметим, что численная симуляция спектральных линий, требующая вычисления либо Бесселовых функций, либо интегралов, аналогично [ $\left.{ }^{8}\right]$ является трудоемкой и не очень

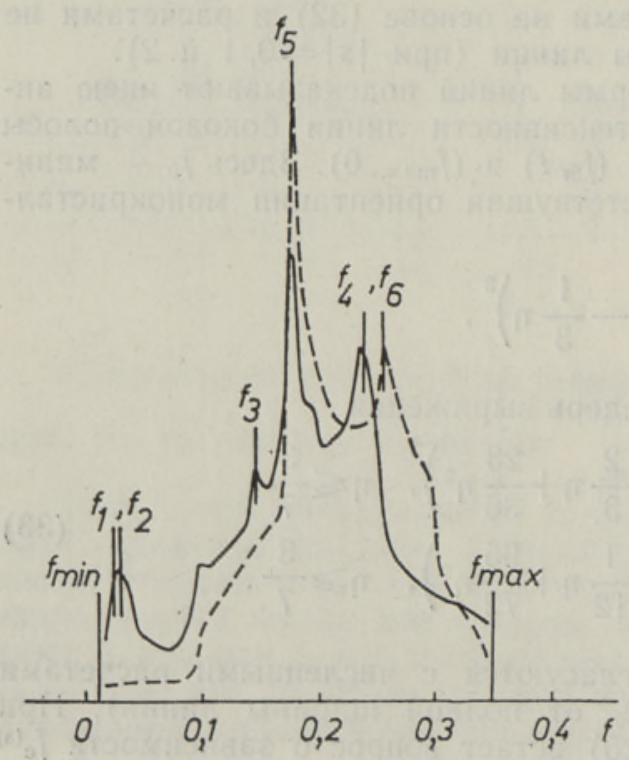
удобной процедурой. Точная алгебраическая функция формы линии $I(f)$ недоступна. В некоторых случаях, по-видимому, допустимо использование приближенной функции $I(f)$. Одним из возможных путей получения приближенной функции формы линии является следующий: на основе уравнений (27) определяется интервал $f^{\prime \prime}(\beta)-f^{\prime}(\beta)$, в интенсивность которого вносят вклад монокристаллы с

Рис. 6. Форма линии первой боковой полосы, рассчитанная на основе уравнений (24) и (25) (сплошная линия) и при помощи приближенной функции $I(f)(34)$ (пунктирная линия), $\eta=0,5$. 
фиксированным углом $\beta$, и пределы ориентационного угла $\beta_{1}$ и $\beta_{2}$, между которыми монокристаллы вносят вклад в интенсивности на фиксированной приведенной частоте. Предполагая равномерное распределение интенсивности по отмеченному частотному интервалу для фунқ:ции формы линии получаем, что

$$
I(f) \sim \int_{\beta_{1}}^{\beta_{2}} \frac{\sin \beta d \beta}{f^{\prime \prime}(\beta)-f^{\prime}(\beta)} .
$$

Отсюда получаем функцию $I(f)$ в аналитическом виде, а на рис, 6 представлен пример ее применения. Существенно отметить, что выражение (34) не отражает максимумы на приведенных частотах $f_{n}, n=$ $=1,2,3$ и 4 , которые обусловлены исключительно неравномерным распределением интенсивностей. Согласие между результатами расчета на основе (34) и численными расчетами тем лучше, чем больше $\eta$.

\section{4. Учет квадрупольного взаимодействия третьего порядка;}

\section{влияние недиагональных элементов $\overline{\tilde{H}}_{Q}^{(1)}$ и диагональных элементов $\overline{\tilde{\mathbf{H}}_{Q}^{(2)}}$}

Ясно, во-первых, что включение недиагональных элементов первого приближения $\left(\overline{\tilde{\mathbf{H}}}_{Q}^{(1)}\right)$ и диагональных элементов второго приближения $\left(\overline{\tilde{\mathbf{H}}}_{Q}^{(2)}\right)$ сопровождается увеличением числа гармоник в (1) с 4 до 6. Кроме того, амплитуды первых четырех гармоник уже нельзя точно описать уравнениями (15)-(17), и последние требуют дополнения. Следовательно,такая коррекция ведет к перераспределению интенсивности любого перехода каждого монокристалла между основной и боковыми линиями вращения. Все же амплитуды добавочных гармоник, так и изменение в (17), примерно в $\frac{\omega_{Q}}{\omega_{L}}$ раз меньше вкладов от диагональных элементов $\overline{\tilde{H}}_{Q}^{(1)}$. Учитывая (23), ранее уже пренебрегли последними при определении распределения интенсивностей. Поэтому утверждение о том, что рассматриваемая поправка практически не изменяет распределения интенсивностей, кажется оправданным.

Что касается положения линий произвольного монокристалла образца, то здесь такая поправка является существенной. Действительно, из (11) видно, что линии от симметричных переходов $m \leftrightarrow m-1$ и $-(m-1) \leftrightarrow-m$ любого монокристалла в общем случае не совпадают. Так как $V_{i}, i=0, \pm 1, \pm 2\left[{ }^{6}\right]$, являются функциями углов ориентации, то величина расщепления различна для различных монокристаллов. Поэтому порошковые полосы от симметричных переходов никогда не расщепляются полностью - в случайным образом подготовленном образце всегда существуют монокристаллы с нулевым расщеплением линий от симметричных переходов.

Расчет (11) показывает, что поправка второго приближения не сворачивает $\beta$-интервалов различных $f$, а сдвигает их монотонно (рис. 7 ), т. е. эта поправка не обусловливает добавочных мақсимумов на линии боковых полос порошка. 


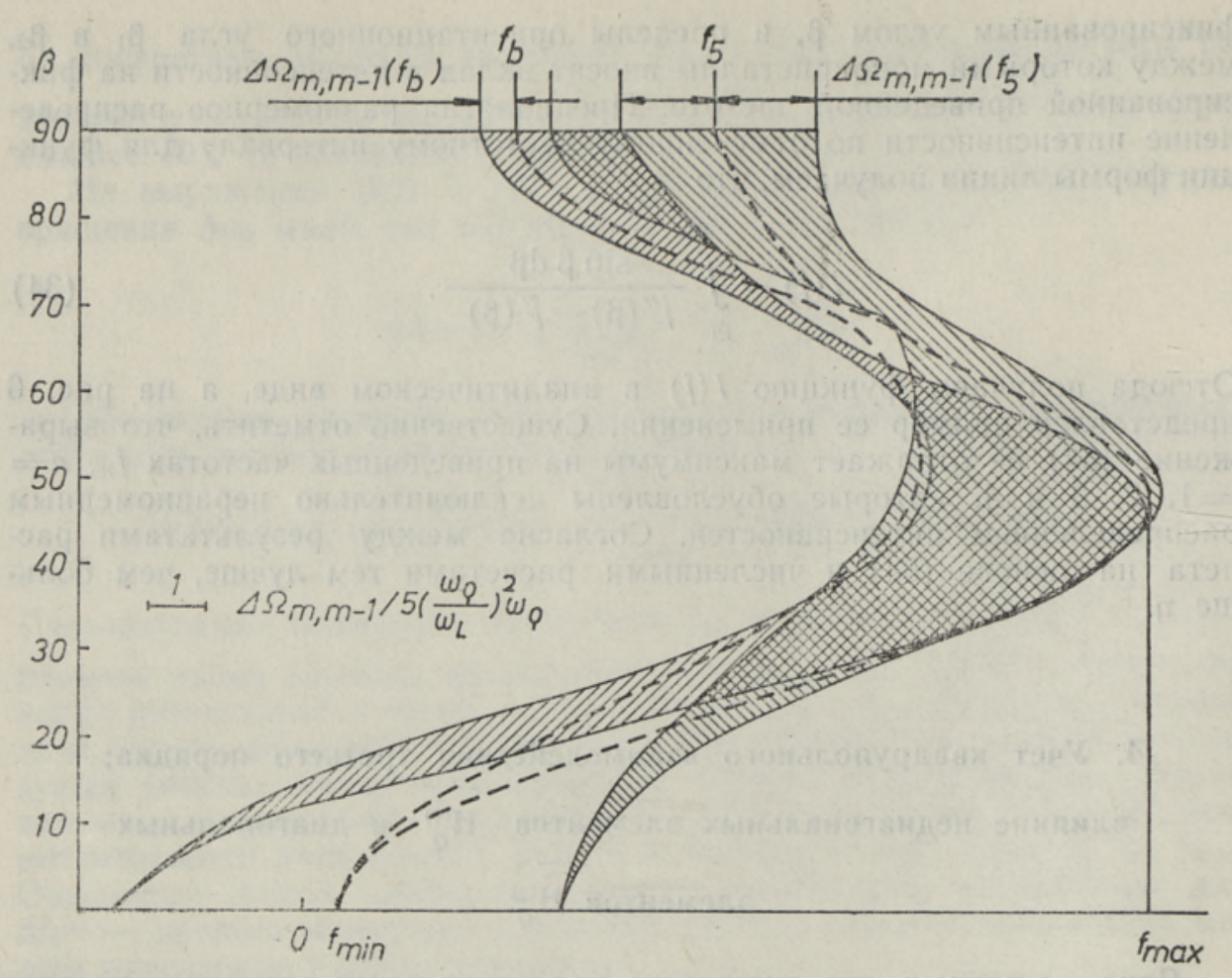

Рис. 7. Области определения ориентационного угла $\beta$ монокристаллов образца, вносящих вклад в интенсивность спектральной линии на фиксированной частоте $f$ от симметрических переходов $m \leftrightarrow m-1$ и $-(m-1) \leftrightarrow-m$ с учетом поправки (11) (сплошная линия) и без учета (11) (пунктирная линия). $I=\frac{9}{2}, \quad m=\frac{5}{2}, \quad \eta=\frac{1}{2}$.

Для величины расщепления главного максимума $f_{5}(28)$ из (11) получаем следующие выражения:

$$
\begin{aligned}
\Delta \Omega_{m, m-1}\left(f_{5}\right) & =\frac{1}{108}\left(\frac{\omega_{Q}}{\omega_{L}}\right)^{2} \omega_{Q}(2 m-1) \times \\
& \times\left\{\left[-33-21 \eta+61 \eta^{2}-7 \eta^{3}\right] I(I+1)+\right. \\
& +\frac{3}{5}\left[45+33 \eta-89 \eta^{2}+11 \eta^{3}\right] m(m-1)+ \\
& \left.+\left[54+42 \eta-110 \eta^{2}+14 \eta^{3}\right]\right\}, \quad \eta \leqslant \frac{3}{7} \\
\Delta \Omega_{m, m-1}\left(f_{5}\right) & =\frac{1}{189}\left(\frac{\omega_{Q}}{\omega_{L}}\right)^{2} \omega_{Q}(2 m-1) \times \\
& \times\left\{16\left(-\frac{177}{49}+\eta^{2}\right) I(I+1)+\right. \\
& +\frac{20}{3}\left(\frac{999}{49}-7 \eta^{2}\right) m(m-1)+ \\
& \left.+\left(\frac{4707}{49}-19 \eta^{2}\right)\right\}, \quad \eta \geqslant \frac{3}{7} .
\end{aligned}
$$


Принимая в качестве условия гарантии наблюдения в эксперименте расщепления главных максимумов симметричных переходов ситуацию, в которой $f$-интервалы, соответствующие $\beta=90^{\circ}$, не перекрываются,

$$
\left|N_{m, m-1} \frac{\omega_{Q}}{\omega_{L}} \omega_{Q}\left(f_{5}-f_{b}\right)\right|<\left|\Delta \Omega_{m, m-1}\left(f_{b}\right)+\Delta \Omega_{m, m-1}\left(f_{5}\right)\right|,
$$

получаем в случае $I=\frac{9}{2}, \eta=\frac{1}{2}$, следующие пороги: $\left|\frac{\omega_{Q}}{\omega_{L}}\right|>6 \cdot 10^{-2}$, $3 \cdot 10^{-3}, 6 \cdot 10^{-2}$ и $8 \cdot 10^{-2}$ соответственно для переходов с $m=\frac{3}{2}, \frac{5}{2}, \frac{7}{2}$ и $\frac{9}{2}$.

В заключение отметим, что рассматриваемая поправка не изменяет положения центра тяжести составной полосы обоих симметричных переходов.

\section{5. Заключение}

ЯМР МВ квадрупольных ядер порошковых образцов с успехом используется для изучения структуры твердых тел. Однако при интерпретации полученных спектров всегда возникает проблема различения с максимально доступной точностью квадрупольного и химического сдвигов.

Оказывается, что для этой цели даже в случае полуцелого спина целесообразно использовать не только центральный, но и один из сателлитных переходов (особенно тогда, когда $I>\frac{3}{2}$ ), а именно, переход с наименьшими значениями параметров квадрупольного сдвига (3) и ширины линии (4). Например, если $I=\frac{5}{2}$ и $I=\frac{9}{2}$, то параметр ширины линии центрального перехода соответственно в 3,4 и 18 раз больше того же параметра дважды симметричного перехода $\left(\frac{3}{2} \leftrightarrow \frac{1}{2}\right.$ и $\left.\frac{5}{2} \leftrightarrow \frac{3}{2}\right)$

Из-за существования квадрупольного взаимодействия первого порядка в спектрах ЯМР МВ нецентральных переходов полуцелого и любых переходов целого спинов появляется множество интенсивных боковых полос вращения. Именно боковые полосы малого порядка отмеченных переходов могут быть использованы для наиболее точной интерпретации спектров в этих случаях.

В настоящей статье анализируются формы линий указанных выше боковых полос. Выводятся приближенные выражения, определяющие положения их сингулярных точек и центров тяжести. Оценивается зависимость положения центра тяжести боковой полосы от малых изменений скорости вращения образца и величина расщепления главных максимумов линий симметричных переходов, обусловленная вторым приближением по $\omega_{Q} / \omega_{L}$. Сравнение, с одной стороны, с численными расчетами и, с другой, с экспериментом демонстрирует хорошую достоверность, а также пределы погрешностей полученных приближенных выражений.

Выведенные спектральные параметры (3), (4) и уравнения особых точек и центра тяжести линии можно с успехом использовать при выборе перехода, наиболее выгодного для регистрации и интерпретации спектра ЯМР МВ квадрупольного ядра порошкового образца. 
Автор выражает искреннюю благодарность профессору Э. Липпмаа за многочисленные обсуждения в ходе работы, А. Самосону, любезно предоставившему неопубликованные экспериментальные данные (рис. 4), которые подкрепляют результаты настоящей теории, и В. Салум за помощь при машинных расчетах,

\section{Л И ТЕ Р А Т У Р А}

1. Eckman, R., Alla, M., Pines, A. J. Magn. Res., 41, 440-446 (1980).

2. Samoson, A. Chem. Phys. Lett., 119, 29-32 (1985); а также устно описанный спектр.

3. Lippmaa, E., Samoson, A., Mägi, M. J. Am. Chem. Soc., 108, 1730-1735 (1986).

4. Kundla, E., Samoson, A., Lippmaa, E. Chem. Phys. Lett., 83, 229-232 (1981).

5. Samoson, A., Kundla, E., Lippmaa, E. J. Magn. Res., 49, 350-357 (1982).

6. Kundla, E. Proc. Acad. Sci. ESSR. Phys. Math., 34, № 1, 68-76 (1985).

7. Mehring, M. Principles of High Resolution NMR in Solids. Berlin-Heidelberg-New York, Springer Verlag, 1983.

8. Herzfeld, J., Berger, A. E. J. Chem. Phys., 73, 6021-6030 (1980).

Ннститут химической и биологической физики Академии наук Эстонской ССР
Поступила в редакцию $20 /$ I 1987

\section{E. KUNDLA}

\section{KVADRUPOOLTUUMADE TMR POORLEVATES PULBRITES: POOLEARVULISTE SPINNIDE MITTETSENTRAALSETE JA TÄISARVULISTE SPINNIDE MIS TAHES OLEMINEKUTE SPEKTRID}

Pöörlevatest pulbrilistest proovidest saadud poolearvuliste spinnidega kvadrupooltuumade mittetsentraalsete üleminekute ja täisarvuliste spinnide mis tahes üleminekute TMR spektrid sisaldavad lisaks keskribale palju intensiivseid külgribasid. Kvadrupooltuuma spektrijoonte summaarse nihke lahutamine tuuma isotroopseks keemiliseks nihkeks ja kvadrupoolseks nihkeks õnnestub sageli kõige täpsemini just nimelt esimesi külgribasid kasutades. Töös on esitatud külgribade singulaarsete punktide ja raskuskeskme ligikaudsed avaldised ning uuritud raskuskaskme stabiilsust pöörlemissageduse väikeste muutuste suhtes. On esitatud spektraalparameetrite avaldised, mis aitavad valida võimalike üleminekute hulgast väikseima kvadrupoolse nihke ja spektrijoone laiusega ülemineku.

Selgub, et sümmeetrilistele üleminekutele $m \leftrightarrow m-1$ ja $\neg(m-1) \leftrightarrow-m$ vastavad külgribad kattuvad täpselt vvaid esimeses lähenduses $\omega_{Q} / \omega_{L}$ järgi. On hinnatud nendele üleminekutele vastavate külgribade peamaksimumi lôhenemise suurust $\omega_{Q} / \omega_{L}$ järgi teises lähenduses.

\section{E. KUNDLA}

\section{MAS-NMR OF QUADRUPOLAR NUCLEI IN POWDERS: HALFINTEGER SPIN NONCENTRAL AND INTEGER SPIN ARBITRARY TRANSITION SPECTRA}

Halfinteger quadrupolar spin noncentral transition and integer spin arbitrary transition MAS-NMR powder spectra contain, besides the central band, a large number of intensive rotational sidebands. Low-order sidebands can in many cases be used for precise separation of the quadrupolar and isotropic chemical shifts of quadrupolar nuclei. In this paper approximate formulas for the singular points and the center of gravity of sideband lineshapes are presented. Stability of the center of gravity under small changes in the rotation rate are pointed out.

Spectral parameters of transitions with the smallest quadrupolar shift and line spread are given. It appears that the sidebands of symmetric $(m \leftrightarrow m-1$ and $-(m-1) \leftrightarrow-m)$ transitions coincide exactly only in the first approximation and the magnitude of splitting of the composite sideband main maximum is estimated in the second approximation by $\omega_{Q} / \omega_{L}$. 